\title{
DNA Methylation Status of PAX1 and ZNF582 in Esophageal Squamous Cell Carcinoma
}

\author{
Jin Huang ${ }^{1,2,+}$, Guo Wang ${ }^{1,2,+}$, Jie Tang ${ }^{1,2}$, Wei Zhuang ${ }^{3}$, Li-Ping Wang ${ }^{4}$, Yu-Ligh Liou ${ }^{1,2,5}$, \\ Ying-Zi Liu ${ }^{1,2}$, Hong-Hao Zhou ${ }^{1,2, *}$ and Yuan-Shan Zhu 1,2,* \\ 1 Department of Clinical Pharmacology, Xiangya Hospital, Central South University, \\ Changsha 410078, Hunan, China; huangjin879288@163.com (J.H.); wangguo32@126.com (G.W.); \\ daratj@163.com (J.T.); yuligh@istat.com.tw (Y.-L.L.); yzliu2005@163.com (Y.-Z.L.) \\ 2 Institute of Clinical Pharmacology, Central South University, Hunan Key Laboratory of Pharmacogenetics, \\ Changsha 410078, Hunan, China \\ 3 Department of Cardiovascular \& Thoracic Surgery, Xiangya Hospital, Central South University, \\ Changsha 410008, Hunan, China; zhuangweihy@sina.com \\ 4 Department of Clinical Oncology, The First People's Hospital of Chenzhou, \\ Chenzhou 423000, Hunan, China; wlpwlp2005@126.com \\ 5 iStat Biomedical Co. Ltd., Taipei 221, Taiwan \\ * Correspondence: hhzhou2003@163.com (H.-H.Z.); 212133@csu.edu.cn (Y.-S.Z.); \\ Tel.: +86-731-848-05380 (H.-H.Z. \& Y.-S.Z.) \\ + These authors contributed equally to this work.
}

Academic Editor: William Chi-shing Cho

Received: 29 September 2016; Accepted: 10 January 2017; Published: 22 February 2017

\begin{abstract}
Hypermethylation of specific gene promoters is an important mechanism of carcinogenesis. A high frequency of promoter methylation of PAX1 and ZNF582 genes has been detected in cervical cancer. In the present study, we investigated the methylation status of PAX1 and ZNF582 genes in esophageal squamous cell carcinoma (ESCC) tissues. Tumor and paracancerous tissues were obtained from 14 ESCC patients. Genomic DNA was extracted from both tumor and paracancerous tissues, and the concentration of DNA were determined. DNA methylation analysis of PAX1 and ZNF582 genes was carried out using quantitative methylation-specific PCR. To assess the diagnostic performance of the two methylated genes for cancer detection, receiver operating characteristic (ROC) curves were generated. Sensitivities and specificities were tested at cut-offs obtained from the ROC curves. The methylation levels of both PAX1 and ZNF582 genes were significantly higher in tumor tissues compared to non-tumor paracancerous tissues. The methylation rates of PAX1 and ZNF582 in ESCC tumor and paracancerous tissues were $100 \%$ and $21.4 \%(p=0.006), 85.7 \%$ and $0 \%(p<0.001)$, respectively. The sensitivities and specificities of PAX1 and ZNF582 methylation for the detection of cancer were $100 \%$ and $85.7 \%$, and $78.6 \%$ and $100 \%$, respectively. The DNA methylation levels and frequencies of PAX1 and ZNF582 genes were markedly higher in ESCC tumor tissues compared to those in paracancerous tissues. Moreover, the conclusions were verified by using The Cancer Genome Atlas (TCGA) datasets. DNA methylation status of these two genes showed a relatively good sensitivity and specificity for the detection of ESCC tumors. This data suggests that DNA methylation testing holds a great promise for ESCC screening and warrants further prospective population-based studies.
\end{abstract}

Keywords: esophageal squamous cell carcinoma; DNA methylation; PAX1; ZNF582

\section{Introduction}

Esophageal cancer (EC) is one of the most aggressive cancers. The incidence and mortality of esophageal cancer are ranked respectively at the ninth and eighth of malignant tumors worldwide [1]. 
There are two main histological EC types: squamous cell carcinoma (SCC) and adenocarcinoma (AC). These two types have distinct pathogenesis and clinical outcomes. Regional variation in both the incidence and prevalence of common EC types is observed in the world. EC has a high incidence and mortality in China, where it accounts for 70\% of the EC cases worldwide, and ranks at the fourth most common cause of cancer related death. More than $90 \%$ of patients with EC in China are esophageal squamous cell carcinoma (ESCC). Although significant advances have been made in the diagnosis and therapy of ESCC in the last decades, the overall five-year survival rate for advanced EC is only around $10 \%$ due to difficulty in early detection at asymptomatic stage, limited therapeutic weapons and poor prognosis. On the other hand, the five-year survival rate may reach up to $90 \%$ if the disease is diagnosed early in the asymptomatic stage. Thus, the understanding of molecular alterations in EC development and the identification of molecular biomarker(s) for EC early detection are crucial in improving EC clinical outcomes and patients' survival.

As a primary form of epigenetic inheritance, DNA methylation has been extensively studied and widely used for tumor classification, early detection, therapeutic target, and predictive biomarker of metastasis and recurrence. The hypermethylation of $\mathrm{CpG}$ islands in the promoter region of tumor suppressor genes, a key mechanism in tumorigenesis, could impede gene transcription and result in a decrease or loss of gene function, a key mechanism in tumorigenesis [2-4]. Similar to other cancers, epigenetic silencing of tumor suppressor genes by promoter hypermethylation is a common molecular alteration in ESCC [5-12]. Methylation of CPG islands in the tumor suppressor genes prevents the binding of transcription factors to the corresponding DNA response elements, resulting in a decrease in gene transcription, and ultimately, a loss of tumor suppressing function, leading to an uncontrolled cell growth and tumor development. It has been shown that aberrant methylation of some tumor suppressor genes such as PTEN, SFRP1, RASSF1A, DAPK, RUNX3, UCHL1, CDH1, p16INK, FHIT, APC and MGMT [13-18] occurs frequently in esophageal cancer. It is therefore the case that alterations in DNA methylation of specific genes may be a useful biomarker for early esophageal cancer detection. However, whether the promoter methylation status of the PAX1 and ZNF582 genes is associated with ESCC and could be novel biomarkers for early esophageal cancer detection remains to be elucidated.

Paired boxed gene 1 (PAX1) and zinc finger protein 582 (ZNF582) are two tumor suppressor genes. Previous studies have demonstrated that the methylation status of PAX1/ZNF582 may serve as useful biomarkers for the detection of cervical cancer $[19,20]$. Huang et al. have reported that DNA methylation of PAX1 gene is a prognostic indicator for oral squamous cell carcinoma [21]. ESCC is homologous to cervical and oral squamous cell carcinoma, in which they belong to squamous cell carcinoma and share similar pathological process. Based on these facts, we hypothesized that alterations in DNA methylation of PAX1 and ZNF582 genes were associated with ESCC development and progression, which may serve as a potential biomarker for early ESCC detection.

Using quantitative methylation-specific PCR (qMSP), we demonstrated for the first time that PAX1 and ZNF582 genes were aberrantly methylated in ESCC tumor tissues compared to the paracancerous normal tissues, and the levels of DNA methylation were significantly associated with tumor progression. This data suggests that the methylation status of PAX1 and ZNF582 genes may be a potential biomarker for ESCC detection.

\section{Materials and Methods}

\subsection{Patients and Samples}

This study was approved by the Institutional Review Board of Department of Clinical Pharmacology, Xiangya Hospital, Central South University (registration number: CTXY-150005-2; date of approval: 2015-11-18) and by Chinese Clinical Trial Registry (registration number: ChiCTR-ROB15007486; date of approval: 2015-11-29). Tumor and paracancerous tissues were obtained from 14 patients with ESCC, who had surgery to resect the tumor between May and November 2015. Inclusion criteria were as follows: (a) patients with ESCC who needed surgical resection; and (b) cases 
with a sufficient amount of residual tumor and paracancerous tissues for DNA methylation analysis. All subjects were provided a written informed consent prior to the study. Clinical and pathological features of 14 patients were listed in Table 1. Histopathological examination was performed to characterize the tumor tissues, and three to five tumor samples were taken from each subject. The tumor tissues were defined as the sections next to the tumor diagnosed histopathologically. Paracancerous tissues were taken from surgically dissected tissues approximately $2 \mathrm{~cm}$ away from the defined tumor area without tumor invasion by histopathology. The dissected tumor and paracancerous tissues were further evaluated by histological examination. Also, tumor samples were microdissected to eliminate surrounding non-tumoral tissues or immune infiltrates. Tumor samples were considered pure and acceptable for inclusion in this study.

\subsection{Genomic DNA Preparation}

All tissue samples were fixed in phosphate-buffered saline formalin solution and paraffin-embedded for histological examination. Approximately $300 \mathrm{mg}$ of paraffin-embedded tissues were dissolved in $1 \mathrm{~mL}$ turpentine and vortexed thoroughly. The sample was then centrifuged at $16,000 \times g$ at a bench-top centrifuge for $3 \mathrm{~min}$ and washed with $70 \%$ ethanol 3 times.

Genomic DNA was extracted using iStat Nucleic Acid Extraction kit (iStat Biomedical Co., Ltd., New Taipei City, Taiwan) according to the manufacturer's standard protocol. DNA concentration was determined using a BioSpec-Nano spectrophotometer. Samples with a DNA yield of more than $500 \mathrm{ng}$ were used for subsequent testing.

\subsection{DNA Methylation Determination}

Bisulfite conversion of DNA samples was carried out using the iStat Bisulfite Conversion Kit (iStat Biomedical Co., Ltd., New Taipei City, Taiwan) following the manufacturer's manual. Bisulfited genomic DNA was analyzed for methylation status by BioSpec-Nano spectrophotometer following the standard instructions.

PAX1 DNA Detection Kit and ZNF582 DNA Detection Kit (iStat Biomedical Co., Ltd., New Taipei City, Taiwan)—simplified technologies which were based on TaqMan technologies for qMSP [22,23] - were used for DNA methylation analysis of PAX1 and ZNF582 genes, respectively. PCR analysis was performed in the Lightcycler LC480 real-time PCR system (Roche Applied Science, Penzberg, Germany). The COL2A gene was used as an internal reference and analyzed parallelly with each specimen. The crossing point $(\mathrm{Cp})$ value for $C O L 2 A \leq 35$ was set as the validity indicator for the testing according to the manufacturer's protocol.

qMSP was done in a $20 \mu \mathrm{L}$ reaction containing $2 \mu \mathrm{L}$ of bisulfite converted template gDNA (50 ng), $1 \mu \mathrm{L}$ of $2 \times$ custom detection mix, and $10 \mu \mathrm{L}$ of $2 \times$ custom universal PCR master mix. The reactions were subjected to a pre-incubation at $95^{\circ} \mathrm{C}$ for $10 \mathrm{~min}$, followed by 50 cycles at $95^{\circ} \mathrm{C}$ for $10 \mathrm{~s}$, and annealing and extension at $60^{\circ} \mathrm{C}$ for $40 \mathrm{~s}$. Fluorescence data were collected during the annealing/extension step for the determination of Cp using LC480. For each sample, the PCR allows to detect simultaneously the methylated strands of ZNF582 or PAX1 using a FAM-labeled probe, while a probe labeled with VIC amplifies a CPG free region of the COL2A gene as internal control, therefore normalizing for the DNA quantity. The DNA methylation levels were assessed as methylation index (meth-index) calculated as follows: $10,000 \times 2^{(\mathrm{Cp} \text { value of gene }-\mathrm{Cp} \text { value of COL2A) }}$. DNA sample from CaSki cervical cancer cells was used as a methylation control, while DNA sample from C33A cells was used as a non-methylation control as previously described [24] to ensure the quality of bisulfite conversion and qMSP processing. The methylation index for these cell lines were shown in insets image of Figure 1.

\subsection{Validation Using TCGA Datasets}

The datasets of DNA methylation information of 187 esophageal cancer tissues and 16 normal tissues was obtained from The Cancer Genome Atlas (TCGA) database. DNA methylation information were Level 3 datasets and were obtained through an Infinium HumanMethylation 450 BeadChip. 

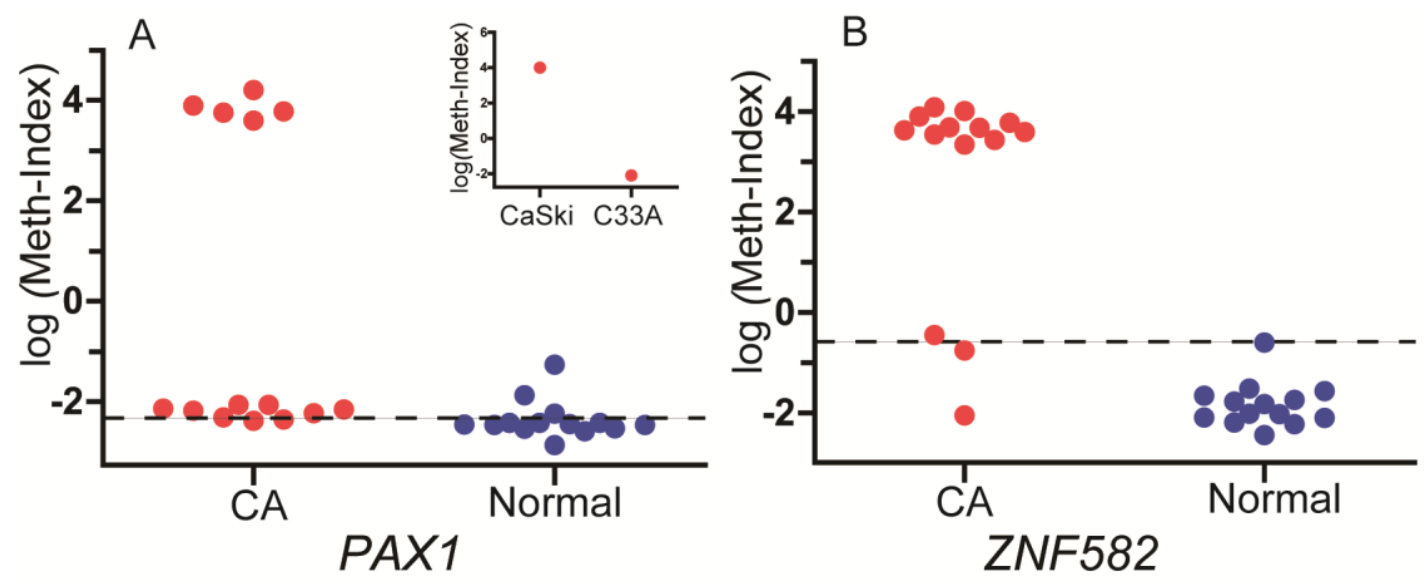

Figure 1. The levels of PAX1 (A) and ZNF582 (B) methylation in cancer (CA) and paracancerous (normal) tissues from 14 esophageal squamous cell carcinoma (ESCC) patients. The level of DNA methylation is presented as log (meth-index). Insets image shows the log (meth-index) of PAX1 for CaSki and C33A cell lines, which were used as methylation and non-methylation controls, respectively. The cut-off value is shown as a dashed line.

\subsection{Statistical Analysis}

To evaluate the diagnostic performance of the two methylated genes for cancer detection, receiver operating characteristic (ROC) curves were generated, and the area under the ROC curve (AUC) was calculated for each gene (Figure 2). The cut-off values for PAX1 and ZNF582 genes were generated from the 14 subjects with methylation results of paracancerous and tumor tissues from the ROC curves, and the sensitivities and specificities were established using cut-off values determined from the ROC curves. $t$-test was used to compare the methylation levels between the ESCC and paracancerous (normal) samples by using TCGA datasets (Figure 3). The correlation between categorical variables was determined with Fisher exact tests (Tables 2 and 3). Mann-Whitney $U$ test was used to analyze the correlation of clinical characteristics with methylation level (Table 4). $p<0.05$ was considered statistically significant. All statistical analysis was performed using SPSS Statistics 19.0 software (IBM Corporation, Armonk, NY, USA). Raw and processed data are stored by the corresponding author of this paper and are available upon request.
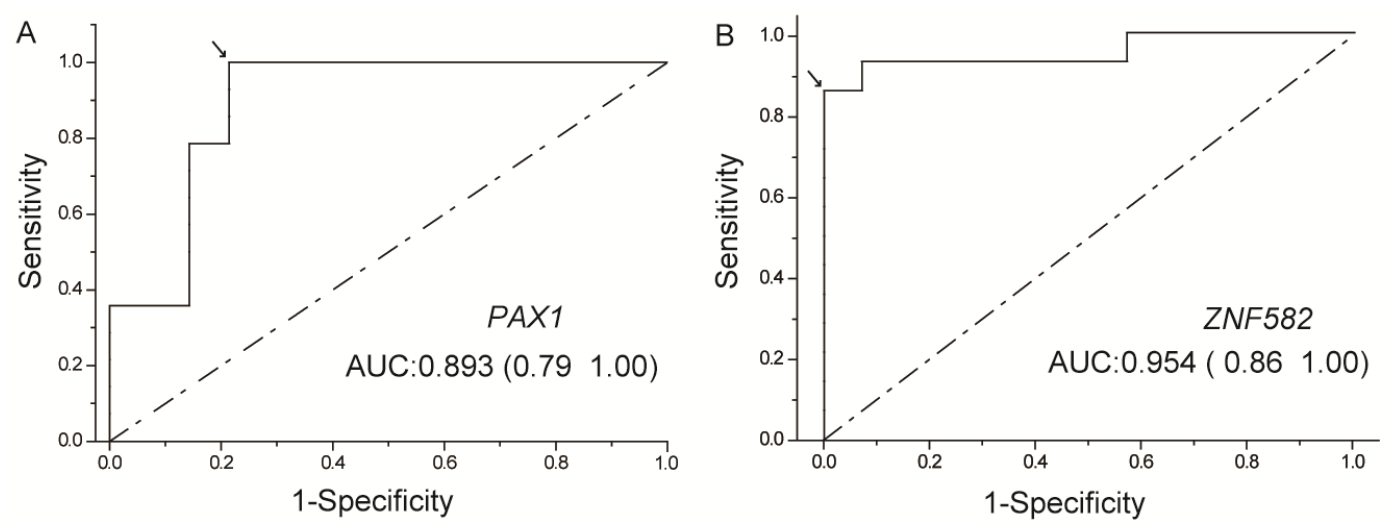

Figure 2. Receiver operating characteristic (ROC) curve analysis of PAX1 (A) and ZNF582 (B). The area under the ROC curve (AUC) of each gene was calculated for the diagnosis of ESCC tumors. The arrow indicates the best sensitivity and specificity. 
Table 1. ESCC tissues of patients with methylation status of PAX1/ZNF582 genes.

\begin{tabular}{|c|c|c|c|c|c|c|c|c|c|c|c|c|c|c|}
\hline \multirow{2}{*}{$\begin{array}{c}\text { Patient } \\
\text { No. }\end{array}$} & \multicolumn{4}{|c|}{ Methylation Status } & \multirow{2}{*}{ Age } & \multirow{2}{*}{ Gender } & \multirow{2}{*}{$\begin{array}{l}\text { TNM } \\
\text { Stage }\end{array}$} & \multirow{2}{*}{$\begin{array}{c}\text { Tumor } \\
\text { Size }\end{array}$} & \multirow{2}{*}{ Tumor HG } & \multirow{2}{*}{$\begin{array}{l}\text { Tumor } \\
\text { Invasion }\end{array}$} & \multirow{2}{*}{$\begin{array}{c}\text { LN } \\
\text { Metastasis }\end{array}$} & \multirow{2}{*}{$\begin{array}{c}\text { DM or } \\
\text { Recurrence }\end{array}$} & \multirow{2}{*}{$\begin{array}{c}\text { Family History } \\
\text { of ESCC }\end{array}$} & \multirow{2}{*}{$\underset{(\mathrm{mg} / \mathrm{mL})}{\mathrm{CEA}}$} \\
\hline & PAX1CA & PAX1P & ZNF582CA & ZNF582P & & & & & & & & & & \\
\hline 1 & + & - & + & - & 47 & Male & $\mathrm{IIb}$ & $<3 \mathrm{~cm}$ & Poor & $\mathrm{T} 2$ & N1 & Negative & Negative & $<9.7$ \\
\hline 2 & + & - & + & - & 74 & Male & IIIc & $3-5 \mathrm{~cm}$ & Well/moderate & $\mathrm{T} 4$ & N1 & Negative & Negative & $<9.7$ \\
\hline 3 & + & + & + & - & 58 & Male & IIIa & $>5 \mathrm{~cm}$ & Well/moderate & $\mathrm{T} 2$ & N2 & Negative & Negative & $<9.7$ \\
\hline 4 & + & - & + & - & 60 & Female & IV & $>5 \mathrm{~cm}$ & Well/moderate & $\mathrm{T} 4$ & N1 & Positive & Negative & $>9.7$ \\
\hline 5 & + & - & + & - & 52 & Male & IIIc & $>5 \mathrm{~cm}$ & Poor & $\mathrm{T} 4$ & N2 & Negative & Negative & $<9.7$ \\
\hline 6 & + & - & + & - & 67 & Male & IIIa & $>5 \mathrm{~cm}$ & Poor & $\mathrm{T} 4$ & No & Negative & Negative & $<9.7$ \\
\hline 7 & + & - & + & - & 59 & Female & ІІа & $>5 \mathrm{~cm}$ & Well/moderate & $\mathrm{T} 3$ & No & Negative & Negative & $<9.7$ \\
\hline 8 & + & + & + & - & 57 & Male & IIIc & $3-5 \mathrm{~cm}$ & Well/moderate & $\mathrm{T} 2$ & N3 & Negative & Negative & $<9.7$ \\
\hline 9 & + & - & + & - & 56 & Male & IV & $3-5 \mathrm{~cm}$ & Poor & $\mathrm{T} 4$ & N2 & Positive & Negative & $>9.7$ \\
\hline 10 & + & - & - & - & 47 & Male & IIIc & $>5 \mathrm{~cm}$ & Poor & $\mathrm{T} 4$ & N1 & Negative & Negative & $>9.7$ \\
\hline 11 & + & - & - & - & 67 & Male & IIIc & $3-5 \mathrm{~cm}$ & Well/moderate & $\mathrm{T} 4$ & N1 & Negative & Negative & $<9.7$ \\
\hline 12 & + & - & + & - & 48 & Male & IIIa & $<3 \mathrm{~cm}$ & Well/moderate & $\mathrm{T} 2$ & N2 & Negative & Negative & $>9.7$ \\
\hline 13 & + & - & + & - & 58 & Female & IIIc & $3-5 \mathrm{~cm}$ & Well/moderate & $\mathrm{T} 2$ & No & Negative & Negative & $<9.7$ \\
\hline 14 & + & + & + & - & 45 & Male & IIIC & $3-5 \mathrm{~cm}$ & Poor & $\mathrm{T} 4$ & N2 & Negative & Negative & $<9.7$ \\
\hline
\end{tabular}

CA: cancer tissue; P: paracancer tissue; DM: distant metastasis; HG: histological grade; LN: lymph node; CEA: carcino-embryonic antigen.

Table 2. Sensitivities, specificities, and AUC of PAX1 and ZNF582 methylation in detecting ESCC tumors $(n=14)$.

\begin{tabular}{|c|c|c|c|c|c|c|c|c|c|}
\hline \multirow{2}{*}{$\begin{array}{l}\text { Detection } \\
\text { Modality }\end{array}$} & \multicolumn{2}{|c|}{ Cancer } & \multicolumn{2}{|c|}{ Paracancer } & \multirow{2}{*}{ Sensitivity \% } & \multirow{2}{*}{ Specificity \% } & \multirow{2}{*}{ AUC } & \multirow{2}{*}{$p$ Value * } & \multirow{2}{*}{$95 \% \mathrm{CI}$} \\
\hline & $\mathrm{U}$ & $\mathbf{M}$ & $\mathbf{U}$ & $\mathbf{M}$ & & & & & \\
\hline PAX1 & 0 & 14 & 11 & 3 & 100.0 & 78.6 & 0.893 & $<0.001$ & $0.764-1.000$ \\
\hline ZNF582 & 2 & 12 & 14 & 0 & 85.7 & 100.0 & 0.954 & $<0.001$ & $0.871-1.000$ \\
\hline
\end{tabular}


Table 3. Association of methylation occurrence frequency of PAX1 and ZNF582 in cancer tissues to clinical characteristic in 14 ESCC patients $(n=14)$.

\begin{tabular}{|c|c|c|c|c|c|}
\hline \multirow{2}{*}{ Characteristics } & \multirow{2}{*}{ Overall Patients $(n=14)$} & \multicolumn{2}{|c|}{ Methylated PAX1 } & \multicolumn{2}{|c|}{ Methylated ZNF582 } \\
\hline & & $n$ & Percentage & $n$ & Percentage \\
\hline Age & & & & & \\
\hline Mean (SD) (range) & $57(8.6)(45-74)$ & & & & \\
\hline$<50$ & 4 & 4 & $100.0 \%$ & 3 & $75.0 \%$ \\
\hline$>50$ & 10 & 10 & $100.0 \%$ & 9 & $90.0 \%$ \\
\hline \multicolumn{6}{|l|}{ Gender } \\
\hline Male & 11 & 11 & $100.0 \%$ & 9 & $81.8 \%$ \\
\hline Female & 3 & 3 & $100.0 \%$ & 3 & $100.0 \%$ \\
\hline \multicolumn{6}{|l|}{ TNM Stage } \\
\hline I/II & 2 & 2 & $100.0 \%$ & 2 & $100.0 \%$ \\
\hline III/IV & 12 & 12 & $100.0 \%$ & 10 & $83.3 \%$ \\
\hline \multicolumn{6}{|l|}{ Tumor size } \\
\hline$<3 \mathrm{~cm}$ & 2 & 2 & $100.0 \%$ & 2 & $100.0 \%$ \\
\hline $3-5 \mathrm{~cm}$ & 6 & 6 & $100.0 \%$ & 5 & $83.3 \%$ \\
\hline$>5 \mathrm{~cm}$ & 6 & 6 & $100.0 \%$ & 5 & $83.3 \%$ \\
\hline \multicolumn{6}{|l|}{ Tumor HG } \\
\hline $\mathrm{W} / \mathrm{M}$ & 8 & 8 & $100.0 \%$ & 7 & $87.5 \%$ \\
\hline Poor & 6 & 6 & $100.0 \%$ & 5 & $83.3 \%$ \\
\hline \multicolumn{6}{|l|}{ Tumor invasion } \\
\hline $\mathrm{T} 1 / 2$ & 5 & 5 & $100.0 \%$ & 5 & $100.0 \%$ \\
\hline $\mathrm{T} 3 / 4$ & 9 & 9 & $100.0 \%$ & 7 & $77.8 \%$ \\
\hline \multicolumn{6}{|l|}{ LN metastasis } \\
\hline No & 3 & 3 & $100.0 \%$ & 3 & $100.0 \%$ \\
\hline $\mathrm{N} 1 / 2 / 3$ & 11 & 11 & $100.0 \%$ & 9 & $81.8 \%$ \\
\hline \multicolumn{6}{|l|}{ DM or recurrence } \\
\hline Negative & 12 & 12 & $100.0 \%$ & 10 & $83.3 \%$ \\
\hline Positive & 2 & 2 & $100.0 \%$ & 2 & $100.0 \%$ \\
\hline \multicolumn{6}{|l|}{ CEA $(\mathrm{ng} / \mathrm{mL})$} \\
\hline$>9.7$ & 4 & 4 & $100.0 \%$ & 3 & $75.0 \%$ \\
\hline$<9.7$ & 10 & 10 & $100.0 \%$ & 9 & $90.0 \%$ \\
\hline
\end{tabular}

DM: distant metastasis; LN: lymph node; HG: histological grade; W/M: well/moderate; CEA: carcinoembryonic antigen.
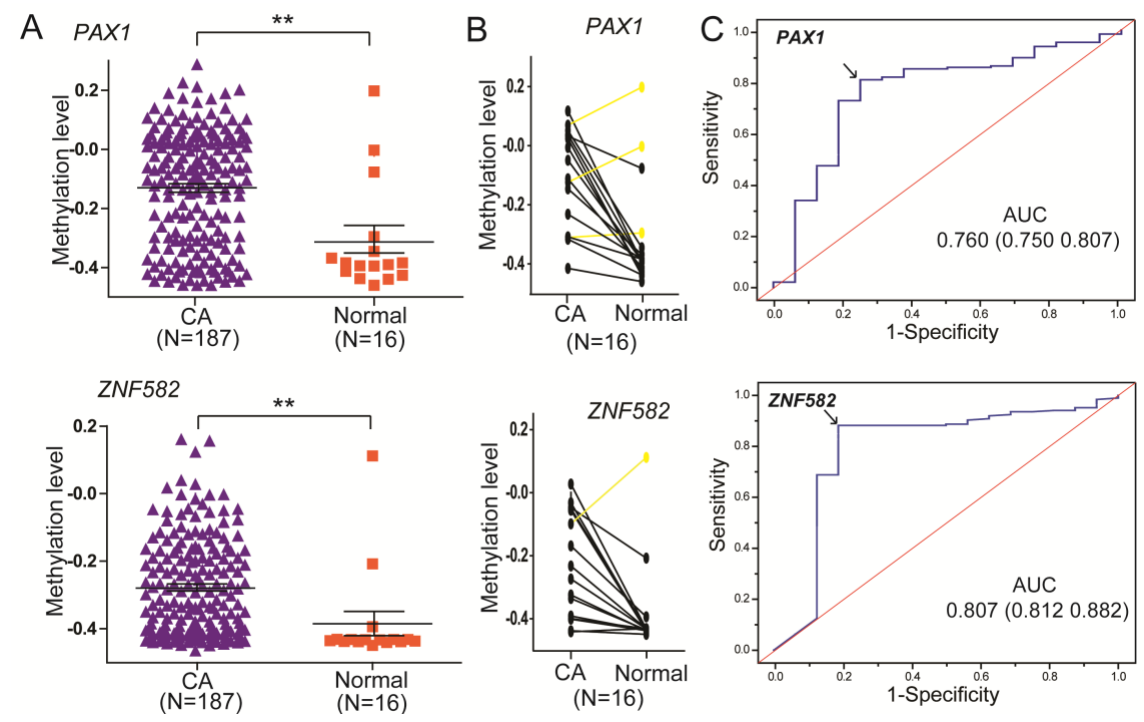

Figure 3. DNA methylation of PAX1 (cg08156066) and ZNF582 (cg11740878) analyzed in esophageal cancer samples using The Cancer Genome Atlas (TCGA) dataset. (A) The methylation level of PAX1 and ZNF582 in 187 esophageal cancer samples and 16 paracancerous samples. ${ }^{* *} p<0.05$ was considered significant; (B) Methylation levels of PAX1 and ZNF582 in 16 esophageal cancer samples and their paired normal samples; (C) ROC curve analysis of PAX1 and ZNF582 for the diagnosis of esophageal cancers from paracancerous tissue. 
Table 4. Correlation between methylation levels of PAX1 and ZNF582 in cancer tissues and clinical characteristics in 14 ESCC patients $(n=14)$.

\begin{tabular}{|c|c|c|c|c|}
\hline \multirow{2}{*}{ Characteristics } & \multicolumn{2}{|c|}{ PAX1 Methylation Level Log (Meth-Index) } & \multicolumn{2}{|c|}{ ZNF582 Methylation Level Log (Meth-Index) } \\
\hline & Mean \pm SD & $p$ Value * & Mean \pm SD & $p$ Value * \\
\hline Age & & 0.620 & & 0.156 \\
\hline$<50$ & $0.763 \pm 3.363$ & & $1.100 \pm 2.788$ & \\
\hline$>50$ & $-0.370 \pm 2.992$ & & $2.279 \pm 2.360$ & \\
\hline Gender & & 0.350 & & 0.755 \\
\hline Male & $0.540 \pm 3.170$ & & $1.851 \pm 2.566$ & \\
\hline Female & $-2.193 \pm 0.162$ & & $2.276 \pm 2.361$ & \\
\hline TNM Stage & & 0.068 & & 0.715 \\
\hline $\mathrm{I} / \mathrm{II}$ & $-2.304 \pm 0.102$ & & $3.469 \pm 0.177$ & \\
\hline III/IV & $0.330 \pm 3.108$ & & $1.688 \pm 2.557$ & \\
\hline Tumor size & & 0.747 & & 0.651 \\
\hline$<5 \mathrm{~cm}$ & $-0.183 \pm 3.172$ & & $1.123 \pm 2.947$ & \\
\hline$>5 \mathrm{~cm}$ & $0.057 \pm 3.109$ & & $2.556 \pm 1.961$ & \\
\hline Tumor HG & & 0.897 & & 0.301 \\
\hline $\mathrm{W} / \mathrm{M}$ & $-0.663 \pm 2.780$ & & $2.131 \pm 2.227$ & \\
\hline Poor & $0.776 \pm 3.376$ & & $1.690 \pm 2.900$ & \\
\hline Tumor invasion & & 0.894 & & 0.423 \\
\hline $\mathrm{T} 1 / 2$ & $-0.938 \pm 2.702$ & & $2.798 \pm 1.823$ & \\
\hline $\mathrm{T} 3 / 4$ & $0.450 \pm 3.217$ & & $1.466 \pm 2.702$ & \\
\hline LN metastasis & & 0.073 & & 0.876 \\
\hline N0 & $-2.275 \pm 0.123$ & & $1.744 \pm 3.282$ & \\
\hline $\mathrm{N} 1 / 2 / 3$ & $0.562 \pm 3.149$ & & $1.996 \pm 2.361$ & \\
\hline DM or recurrence & & 0.411 & & 0.522 \\
\hline $\begin{array}{l}\text { Negative } \\
\text {. }\end{array}$ & $0.315 \pm 3.122$ & & $2.017 \pm 2.512$ & \\
\hline Positive & $-2.210 \pm 0.204$ & & $1.492 \pm 2.746$ & \\
\hline CEA (ng/mL) & & 0.887 & & 0.076 \\
\hline$>9.7$ & $-0.722 \pm 2.883$ & & $0.123 \pm 2.332$ & \\
\hline$<9.7$ & $0.224 \pm 3.174$ & & $2.670 \pm 2.174$ & \\
\hline
\end{tabular}

DM: distant metastasis; LN: lymph node; HG: histological grade; W/M: well/moderate; CEA: carcino-embryonic antigen. * Association analysis for methylation status by Mann-Whitney $\mathrm{U}$ test. $p<0.1$ are indicated in bold.

\section{Results}

\subsection{The Levels and Frequencies of DNA Methylation of PAX1 and ZNF582 Genes in ESCC Tumor and} Paracancerous Tissues

A total of 14 subjects with ESCC were recruited from Xiangya Hospital located in Changsha, China between May and November 2015. The demographic characteristics of these subjects are presented in Tables 1 and 3. There were 11 males and three females with a mean age of 57 (age range 45-74 years old), four of them younger than 50 and 10 of them older than 50 . DNA methylation of PAX1 and ZNF582 genes in both tumor and paracancerous tissues was detected successfully using the current analysis. As shown in Figure 1, the methylation levels of both genes were higher in tumor tissues compared to those in paracancerous tissues. The methylation status of samples and frequencies were determined based on the best meth-index cut-off values calculated using the ROC curves (Figure 2). The cut-off values were shown in Figure 1. Along the lines, log (meth-index) $\geq-2.376$ for PAX1 or $\log$ (meth-index) $\geq-0.597$ for ZNF582 were considered to be methylation positivity. The frequency of PAX1 methylation was $100 \%(14 / 14)$ in the tumor tissues, which is significantly higher than that $(21.4 \%, 3 / 14)$ in the paracancerous tissues. The frequency of ZNF582 methylation in tumor tissues $(85.7 \%, 12 / 14)$ is significantly higher than that $(0 \%, 0 / 14)$ in paracancerous tissues (Table 2$)$.

Additionally, the results were verified using TCGA datasets. As shown in Figure 3A, the methylation levels of both genes were higher in 187 tumor samples compared to 16 paracancerous samples. The frequency of PAX1 methylation was $80.7 \%(151 / 187)$ in the tumor samples, which is significantly higher than that $(25 \%, 4 / 16)$ in the paracancerous samples. The frequency of ZNF582 methylation was $88.2 \%(165 / 187)$ in the tumor samples, which is significantly higher than that $(18.8 \%, 3 / 16)$ in the paracancerous samples. Besides, as shown in Figure 3B, the methylation level of 
PAX1 was higher in tumor samples compared to their paired paracancerous samples in $81.3 \%(13 / 16)$ ESCC patients. The methylation level of ZNF582 was higher in the tumor samples compared to their paired paracancerous samples in $93.8 \%(15 / 16)$ ESCC patients.

\subsection{The Sensitivity and Specificity of DNA Methylation Testing of PAX1 and ZNF582 Genes in Distinguishing ESCC Tumors from Non-Tumor Tissues}

To evaluate the clinical application, ROC curves were generated and the AUC was calculated for both PAX1 and ZNF582 methylation to discriminate tumor from non-tumor tissues (Figure 2). The accuracies of PAX1 and ZNF582 methylation testing were 0.893 and 0.954 , respectively (Table 2). As shown in Figure 2 and Table 2, the sensitivity and specificity of PAX1 methylation testing achieved $100 \%$ and $78.6 \%$ at the best meth-index cut-off value of -2.376 , respectively. At the best meth-index cut-off value of -0.597 , the sensitivity and specificity of ZNF582 methylation testing were $85.7 \%$ and $100 \%$, respectively.

Additionally, as shown in Figure 3C, the results of the accuracies of PAX1 and ZNF582 methylation testing using TCGA datasets were 0.760 and 0.806 , respectively (Table 2). The sensitivity and specificity of PAX1 methylation testing achieved $80.7 \%$ and $75.0 \%$, respectively, and the sensitivity and specificity of ZNF582 methylation testing were $88.2 \%$ and $81.2 \%$, respectively.

\subsection{Association of PAX1 and ZNF582 Methylation to Clinical and Pathological Features of the Patients}

The association of PAX1 and ZNF582 methylation levels to clinical and pathological characteristics was shown in Table 4. Mann-Whitney $U$ test was used to analyze the levels of gene methylation in the different groups. Unfortunately, most $p$ values were above 0.05 due to small sample sizes. However, there is a tendency that PAX1 methylation level was associated with TNM stage and lymph node metastasis with a $p$ value of 0.068 and 0.073 , respectively, while ZNF582 methylation level was associated with carcino-embryonic antigen (CEA) concentration with a $p$ value of 0.076 (Table 4). However, there is no association between methylation occurrence frequency of PAX1 and ZNF582 and any clinical features as shown in Table 3.

\section{Discussion}

DNA methylation plays a crucial role in the regulation of gene expression and is a potential biomarker for cancer detection. Previous studies have shown that the methylation status of PAX1 and ZNF582 genes is a useful testing to distinguish tumor and non-tumor tissues in cervical and oral squamous cell carcinoma $[19,20]$. In the present study, we have for the first time demonstrated that the levels and frequencies of PAX1 and ZNF582 methylation are markedly higher in the tumor tissues compared to non-tumor tissues from ESCC patients, and methylation testing of these two genes has an excellent accuracy and great sensitivity and specificity to detect ESCC tumors, suggesting that PAX1 and ZNF582 methylation testing may be a promising biomarker for the detection of ESCC.

$P A X 1$ gene is a member in the group 1 of the paired box $(P A X)$ family of transcription factors, which has four well-defined groups [25]. Members of the PAX family typically contain a paired box domain and a paired-type homeodomain, and play critical roles in pattern formation during embryogenesis. PAX members have been reported to be overexpressed in several cancers and function in oncogenesis [26-28]. On the other hand, PAX1 and PAX4 are silenced by DNA methylation in ovarian and cervical cancers and in melanoma, and may function as tumor suppressors [29-31]. As a tumor suppressor gene, PAX1 may be involved in carcinogenesis and tumor progression to invasive or aggressive cancers. Previous studies have reported that PAX1 methylation testing is a potential biomarker for the screening of cervical cancer with a sensitivity and specificity greater than $80 \%$ in the detection of grade III or higher cervical intraepithelial neoplasia (CIN3+) lesions [32,33]. The efficacy of PAX1 methylation testing in detecting cervical cancer is significantly improved with a combination of cervical cell cytology or HPV 16, 18 genotyping $[34,35]$. Consistent with the reports in cervical cancers, we observed in the current study that PAX1 methylation testing had a $100 \%$ sensitivity and a $78.6 \%$ 
specificity in the detection of ESCC tumors, indicating that PAX1 methylation is a valuable biomarker for ESCC diagnosis.

ZNF582 (zinc finger protein 582) gene, located at chromosome 19q13.43, encodes a protein, ZNF582, in the KRAB-ZNF family, which contains a KRAB-AB domain and nine zinc-finger motifs [22,36]. Most KRAB-ZNF proteins contain the KRAB-AB domain and bind KRAB-associated protein 1 (KAP1) to co-repress target gene transcription. The KRAB-ZNF proteins including ZNF582 are involved in DNA damage response, proliferation, cell cycle control, and neoplastic transformation [22]. Consistent with the concept that ZNF582 is a tumor suppressor, ZNF582 hypermethylation has been reported in acute myeloid leukemia and various invasive cancers [37]. Liou et al. reported that ZNF582 methylation testing had a $70 \%$ sensitivity and an $82 \%$ specificity for the detection of cervical cancer CIN3+ lesions [37], and a great sensitivity and specificity in the classification of low-grade squamous intraepithelial lesion (LSIL) [20]. In the present study, we demonstrated that ZNF582 gene was much more frequently methylated in ESCC tumor tissues compared to non-tumor paracancerous tissues. ZNF582 methylation testing reaches a 100\% specificity and an $85.7 \%$ sensitivity for the detection of ESCC tumors (see Table 2), suggesting that it is a useful biomarker for screening ESCC. Moreover, a combination of PAX1 and ZNF582 methylation testing could reach a 100\% sensitivity and specificity in the detection of ESCC tumors.

To further evaluate the clinical significance of PAX1 and ZNF582 methylation in ESCC development and progression, the association of PAX1/ZNF582 methylation to the clinicopathological features of ESCC patients was analyzed, as a previous report [38] indicated a significant association between PAX6 methylation and TNM stage of non-small cell lung cancer (NSCLC). In the present study, we did not observe any significant association between PAX1 or ZNF582 methylation status and the clinical features analyzed. However, the associations of PAX1 methylation with TNM stage $(p=0.068)$ and LN metastasis $(p=0.073)$ and ZNF582 methylation with CEA concentration $(p=0.076)$ appear to be interesting. This observation is in agreement with previous studies in cervical cancer $[19,20]$.

Nowadays, a novel method of swallowing a sponge on a string for esophageal cancer detection has been proposed [39-41]. Patients using the cytosponge swallow a small capsule on a string, which expands in their stomach over a few minutes to form a small sponge. The sponge is then gently pulled back out, bringing a sample of cells with it as it comes up through the person's esophagus. The methylation of PAX1 and ZNF582 detected in these cells could act as biomarkers for esophageal cancer screening in the future, which could replace traditional endoscopy as an equally effective but less invasive way of diagnosing for esophageal cancer.

However, it should be noted that there are several limitations in the present study. First, the sample size was small and further studies with a larger sample size are needed to confirm these findings. Second, we could not evaluate the prognostic value of DNA methylation due to the lack of follow-up data. Third, biopsy-matched samples, rather than population-based screening samples, were used to investigate the overall methylation status, precluding the evaluation of its triage potential. Thus, a larger population-based screening or triage trial will determine the actual diagnostic and prognostic performance of DNA methylation in esophageal cancer. Finally, although the two genes used in the current study were selected through extensive literature review, a more comprehensive and unbiased assessment of DNA methylation patterns using genome-wide methylation analysis may provide a better understanding of DNA methylation signatures in ESCC. The elucidation of these DNA methylation signatures will facilitate the development of a more effective strategy for ESCC screening and diagnosis, and accelerate the discovery of potential therapeutic targets for ESCC treatment.

\section{Conclusions}

Our current study demonstrated for the first time that both the levels and frequencies of PAX1 and ZNF582 methylation were greatly higher in the ESCC tumor tissues compared to non-tumor paracancerous tissues. PAX1 and ZNF582 methylation testing has an excellent accuracy, sensitivity and specificity in distinguishing ESCC tumor tissues from non-tumor tissues. The combination of PAX1 and 
ZNF582 methylation testing could reach a 100\% sensitivity and specificity in detecting ESCC tumors, providing a promising biomarker for ESCC screening and diagnosis, although further studies with larger sample size or population-based investigation are necessary to confirm this intriguing finding.

Acknowledgments: The authors are indebted to all the donors whose names were not included in the author list, but who participated in our study. This study was supported in part by the National Natural Science Foundation of China (Grant No. 81673516, 81403018 and 81402968), by the Science and Technology Project of Hunan Province, China (Grant 2013FJ3036), by the Hunan Provincial Natural Science Foundation of China (Grant 2015JJ3169) and by the Special Talents Fund from Central South University of China.

Author Contributions: H.H.Z. and Y.S.Z. designed the study. J.H. and G.W. carried out the molecular experiments and data analysis. L.P.W. contributed in data collection. J.T., Y.Z.L. and Y.L.L. provided technical assistance. J.H., G.W. and Y.S.Z. wrote the manuscript. All authors read and approved the final manuscript.

Conflicts of Interest: iStat Biomedical Co., Ltd. supplied the testing kits used for the project. Y.L.L. is Ph.D. student of the Department of Clinical Pharmacology, Xiangya Hospital, Central South University, China, and an employee of iStat Biomedical Co., Ltd., Taiwan. The other authors declare that they have no conflict of interests.

\section{References}

1. Kuwano, H.; Kato, H.; Miyazaki, T.; Fukuchi, M.; Masuda, N.; Nakajima, M.; Fukai, Y.; Sohda, M.; Kimura, H.; Faried, A. Genetic Alterations in Esophageal Cancer. Surg. Today 2005, 35, 7-18. [CrossRef] [PubMed]

2. Jones, P.A.; Baylin, S.B. The fundamental role of epigenetic events in cancer. Nat. Rev. Genet. 2002, 3, 415-428. [PubMed]

3. Herman, J.; Baylin, S. Gene silencing in cancer in association with promoter hypermethylation. N. Engl. J. Med. 2003, 349, 2042-2054. [CrossRef] [PubMed]

4. Juo, Y.Y.; Johnston, F.M.; Zhang, D.Y.; Juo, H.H.; Wang, H.; Pappou, E.P.; Yu, T.; Easwaran, H.; Baylin, S.; van Engeland, M.; et al. Prognostic Value of CpG Island Methylator Phenotype among Colorectal Cancer Patients: A Systematic Review and Meta-Analysis. Ann. Oncol. 2014, 25, 2314-2327. [CrossRef] [PubMed]

5. Ito, S.; Ohga, T.; Saeki, H.; Nakamura, T.; Watanabe, M.; Tanaka, S.; Kakeji, Y.; Maehara, Y. p53 mutation profiling of multiple esophageal carcinoma using laser capture microdissection to demonstrate field carcinogenesis. Int. J. Cancer 2005, 113, 22-28. [CrossRef] [PubMed]

6. Baylin, S.B.; Herman, J.G. DNA hypermethylation in tumorigenesis: Epigenetics joins genetics. Trends Genet. 2000, 16, 168-174. [CrossRef]

7. Li, J.S.; Ying, J.M.; Wang, X.W.; Wang, Z.H.; Tao, Q.; Li, L.L. Promoter methylation of tumor suppressor genes in esophageal squamous cell carcinoma. Chin. J. Cancer 2013, 32, 3-11. [CrossRef] [PubMed]

8. Liu, C.; Li, N.; Lu, H.; Wang, Z.; Chen, C.; Wu, L.; Liu, J.; Lu, Y.; Wang, F. Circulating SFRP1 promoter methylation status in gastric adenocarcinoma and esophageal square cell carcinoma. Biomed. Rep. 2015, 3, 123-127. [CrossRef] [PubMed]

9. Si, H.X.; Tsao, S.W.; Lam, K.Y.; Srivastava, G.; Liu, Y.; Wong, Y.C.; Shen, Z.Y.; Cheung, A.L. E-cadherin expression is commonly downregulated by $\mathrm{CpG}$ island hypermethylation in esophageal carcinoma cells. Cancer Lett. 2001, 173, 71-78. [CrossRef]

10. Takeno, S.; Noguchi, T.; Fumoto, S.; Kimura, Y.; Shibata, T.; Kawahara, K. E-cadherin expression in patients with esophageal squamous cell carcinoma: Promoter hypermethylation, Snail overexpression, and clinicopathologic implications. Am. J. Clin. Pathol. 2004, 122, 78-84. [CrossRef] [PubMed]

11. Lee, E.J.; Lee, B.B.; Han, J.; Cho, E.Y.; Shim, Y.M.; Park, J.; Kim, D.H. CpG island hypermethylation of E-cadherin (CDH1) and integrin $\alpha 4$ is associated with recurrence of early stage esophageal squamous cell carcinoma. Int. J. Cancer J. Int. Du Cancer 2008, 123, 2073-2079. [CrossRef] [PubMed]

12. Tzao, C.; Hsu, H.S.; Sun, G.H.; Lai, H.L.; Wang, Y.C.; Tung, H.J.; Yu, C.P.; Cheng, Y.L.; Lee, S.C. Promoter methylation of the $h M L H 1$ gene and protein expression of human mutL homolog 1 and human mutS homolog 2 in resected esophageal squamous cell carcinoma. J. Thorac. Cardiovasc. Surg. 2005, 130, 1371.e1-1371.e8. [CrossRef] [PubMed]

13. Long, C.; Yin, B.; Lu, Q.; Zhou, X.; Hu, J.; Yang, Y.; Yu, F.; Yuan, Y. Promoter Hypermethylation of the RUNX3 Gene in Esophageal Squamous Cell Carcinoma. Cancer Investig. 2009, 25, 685-690. [CrossRef] [PubMed]

14. Kuroki, T.; Trapasso, F.; Yendamuri, S.; Matsuyama, A.; Alder, H.; Mori, M.; Croce, C.M. Promoter Hypermethylation of RASSF1A in Esophageal Squamous Cell Carcinoma. Clin. Cancer Res. 2003, 9, 1441-1445. [PubMed] 
15. Vasavi, M.; Kiran, V.; Ravishankar, B.; Prabhakar, B.; Ahuja, Y.R.; Hasan, Q. Microsatellite instability analysis and its correlation with $h M L H 1$ repair gene hypermethylation status in esophageal pathologies including cancers. Cancer Biomark. 2010, 7, 1-10. [CrossRef] [PubMed]

16. Guo, M.; Ren, J.; House, M.G.; Qi, Y.; Brock, M.V.; Herman, J.G. Accumulation of promoter methylation suggests epigenetic progression in squamous cell carcinoma of the esophagus. Clin. Cancer Res. 2006, 12, 4515-4522. [CrossRef] [PubMed]

17. Tonomoto, Y.; Tachibana, M.; Dhar, D.K.; Onoda, T.; Hata, K.; Ohnuma, H.; Tanaka, T.; Nagasue, N. Differential Expression of RUNX Genes in Human Esophageal Squamous Cell Carcinoma: Downregulation of RUNX3 Worsens Patient Prognosis. Oncology 2008, 73, 346-356. [CrossRef] [PubMed]

18. Kuo, I.Y.; Chang, J.M.; Jiang, S.S.; Chen, C.H.; Chang, I.S.; Sheu, B.S.; Lu, P.J.; Chang, W.L.; Lai, W.W.; Wang, Y.C. Prognostic CpG methylation biomarkers identified by methylation array in esophageal squamous cell carcinoma patients. Int. J. Med. Sci. 2014, 11, 779-787. [CrossRef] [PubMed]

19. Lai, H.C.; Ou, Y.C.; Chen, T.C.; Huang, H.J.; Cheng, Y.M.; Chen, C.H.; Chu, T.Y.; Hsu, S.T.; Liu, C.B.; Hung, Y.C.; et al. PAX1/SOX1 DNA methylation and cervical neoplasia detection: A Taiwanese Gynecologic Oncology Group (TGOG) study. Cancer Med. 2014, 3, 1062-1074. [CrossRef] [PubMed]

20. Lin, H.; Chen, T.C.; Chang, T.C.; Cheng, Y.M.; Chen, C.H.; Chu, T.Y.; Hsu, S.T.; Liu, C.B.; Yeh, L.S.; Wen, K.C.; et al. Methylated ZNF582 gene as a marker for triage of women with Pap smear reporting low-grade squamous intraepithelial lesions-A Taiwanese Gynecologic Oncology Group (TGOG) study. Gynecol. Oncol. 2014, 135, 64-68. [CrossRef] [PubMed]

21. Huang, Y.K.; Peng, B.Y.; Wu, C.Y.; Su, C.T.; Wang, H.C.; Lai, H.C. DNA methylation of PAX1 as a biomarker for oral squamous cell carcinoma. Clin. Oral Investig. 2014, 18, 801-808. [CrossRef] [PubMed]

22. Huang, R.L.; Chang, C.C.; Su, P.H.; Chen, Y.C.; Liao, Y.P.; Wang, H.C.; Yo, Y.T.; Chao, T.K.; Huang, H.C.; Lin, C.L.; et al. Methylomic analysis identifies frequent DNA methylation of zinc finger protein 582 (ZNF582) in cervical neoplasms. PLOS ONE 2012, 7, e41060. [CrossRef] [PubMed]

23. Lai, H.C.; Lin, Y.W.; Huang, T.H.; Yan, P.; Huang, R.L.; Wang, H.C.; Liu, J.; Chan, M.W.; Chu, T.Y.; Sun, C.A.; et al. Identification of novel DNA methylation markers in cervical cancer. Int. J. Cancer 2008, 123, 161-167. [CrossRef] [PubMed]

24. Ki, E.Y.; Lee, K.H.; Hur, S.Y.; Rhee, J.E.; Kee, M.K.; Kang, C.; Park, J.S. Methylation of Cervical Neoplastic Cells Infected With Human Papillomavirus 16. Int. J. Gynecol. Cancer 2015, 26, 176-183. [CrossRef] [PubMed]

25. Robson, E.J.D.; He, S.J.; Eccles, M.R. A PANorama of PAX genes in cancer and development. Nat. Rev. Cancer 2006, 6, 52-62. [CrossRef] [PubMed]

26. Lang, D.; Powell, S.K.; Plummer, R.S.; Young, K.P.; Ruggeri, B.A. PAX genes: Roles in development, pathophysiology, and cancer. Biochem. Pharmacol. 2007, 73, 1-14. [CrossRef] [PubMed]

27. Galindo, R.L.; Allport, J.A.; Olson, E.N. A Drosophila model of the rhabdomyosarcoma initiator PAX7-FKHR. Proc. Natl. Acad. Sci. USA 2006, 103, 13439-13444. [CrossRef] [PubMed]

28. Abdallah, S.; Wolfram, J.; Meinrad, B. Oncogenic role of PAX5 in the T-lymphoid lineage upon ectopic expression from the immunoglobulin heavy-chain locus. Blood 2008, 109, 281-289.

29. Chang, C.C.; Huang, R.L.; Wang, H.C.; Liao, Y.P.; Yu, M.H.; Lai, H.C. High methylation rate of $L M X 1 A$, NKX6-1, PAX1, PTPRR, SOX1, and ZNF582 genes in cervical adenocarcinoma. Int. J. Gynecol. Cancer 2014, 24, 201-209. [CrossRef] [PubMed]

30. Huang, T.H.; Lai, H.C.; Liu, H.W.; Lin, C.J.; Wang, K.H.; Ding, D.C.; Chu, T.Y. Quantitative analysis of methylation status of the PAX1 gene for detection of cervical cancer. Int. J. Gynecol. Cancer 2010, 20, 513-519. [CrossRef] [PubMed]

31. Hata, S.; Hamada, J.; Maeda, K.; Murai, T.; Tada, M.; Furukawa, H.; Tsutsumida, A.; Saito, A.; Yamamoto, Y.; Moriuchi, T. PAX4 has the potential to function as a tumor suppressor in human melanoma. Int. J. Oncol. 2008, 33, 1065-1071. [PubMed]

32. Kan, Y.Y.; Liou, Y.L.; Wang, H.J.; Chen, C.Y.; Sung, L.C.; Chang, C.F.; Liao, C.I. PAX1 methylation as a potential biomarker for cervical cancer screening. Int. J. Gynecol. Cancer 2014, 24, 928-934. [CrossRef] [PubMed]

33. Chang, C.C.; Huang, R.L.; Liao, Y.P.; Su, P.H.; Hsu, Y.W.; Wang, H.C.; Tien, C.Y.; Yu, M.H.; Lin, Y.W.; Lai, H.C. Concordance analysis of methylation biomarkers detection in self-collected and physician-collected samples in cervical neoplasm. BMC Cancer 2015, 15, 1-9. [CrossRef] [PubMed] 
34. Steenbergen, R.D.M.; Snijders, P.J.F.; Heideman, D.A.M.; Meijer, C.J.L.M. Clinical implications of (epi)genetic changes in HPV-induced cervical precancerous lesions. Nat. Rev. Cancer 2014, 14, 395-405. [CrossRef] [PubMed]

35. Clarke, M.A.; Wentzensen, N.; Mirabello, L.; Ghosh, A.; Wacholder, S.; Harari, A.; Lorincz, A.; Schiffman, M.; Burk, R.D. Human papillomavirus DNA methylation as a potential biomarker for cervical cancer. Cancer Epidemiol. Biomark. Prev. 2012, 21, 2125-2137. [CrossRef] [PubMed]

36. Urrutia, R. KRAB-containing zinc-finger repressor proteins. Genome Biol. 2003, 4, 231. [CrossRef] [PubMed]

37. Liou, Y.L.; Zhang, Y.; Liu, Y.; Cao, L.; Qin, C.Z.; Zhang, T.L.; Chang, C.F.; Wang, H.J.; Lin, S.Y.; Chu, T.Y.; et al. Comparison of HPV genotyping and methylated ZNF582 as triage for women with equivocal liquid-based cytology results. Clin. Epigenet. 2015, 7, 1-9. [CrossRef] [PubMed]

38. Zhang, X.; Yang, X.; Wang, J.; Liang, T.; Gu, Y.; Yang, D. Down-regulation of PAX6 by promoter methylation is associated with poor prognosis in non small cell lung cancer. Int. J. Clin. Exp. Pathol. 2015, 8, 11452-11457. [PubMed]

39. Oh, K.B.; Mar, W.; Kim, S.; Kim, J.Y.; Lee, T.H.; Kim, J.G.; Shin, D.; Sim, C.J.; Shin, J. Antimicrobial activity and cytotoxicity of bis(indole) alkaloids from the sponge Spongosorites sp. Biol. Pharm. Bull. 2006, 29, 570-573. [CrossRef] [PubMed]

40. Benaglia, T.; Sharples, L.D.; Fitzgerald, R.C.; Lyratzopoulos, G. Health benefits and cost effectiveness of endoscopic and nonendoscopic cytosponge screening for Barrett's esophagus. Gastroenterology 2013, 144, 62-73. [CrossRef] [PubMed]

41. Kadri, S.; Lao-Sirieix, P.; Fitzgerald, R.C. Developing a nonendoscopic screening test for Barrett's esophagus. Biomark. Med. 2011, 5, 397-404. [CrossRef] [PubMed]

(C) 2017 by the authors. Licensee MDPI, Basel, Switzerland. This article is an open access article distributed under the terms and conditions of the Creative Commons Attribution (CC BY) license (http:/ / creativecommons.org/licenses/by/4.0/). 